\title{
Intranasal Photobiomodulation Therapy for Brain Conditions: A Review
}

Shin Hyuk Yoo ${ }^{1,2}$

${ }^{1}$ Department of Otorhinolaryngology, Dankook University College of Medicine, Cheonan, Korea

${ }^{2}$ Beckman Laser Institute, Cheonan, Korea
Received July 23, 2021

Accepted August 31, 2021

\section{Correspondence}

Shin Hyuk Yoo

Department of Otorhinolaryngology, Dankook University College of Medicine, 119 Dandae-ro, Dongnam-gu, Cheonan 31116, Korea

Tel.: +82-41-550-3933

Fax: +82-41-556-1090

E-mail: shyoomddamail.com

(c) Korean Society for Laser Medicine and Surgery

(c) This is an open access article distributed under the terms of the Creative Commons Attribution NonCommercial License (http://creativecommons.org/ licenses/by-nc/4.0) which permits unrestricted noncommercial use, distribution, and reproduction in any medium, provided the original work is properly cited.
The effects of low-level laser irradiation on cells and tissues, known as photobiomodulation therapy (PBMT), are the basis of photomedicine. Several investigations have evaluated the therapeutic effects of PBMT for neuronal regeneration and differentiation in animal models and humans. Recently, intranasal PBMT (iN-PBMT) has shown potential as a treatment method for neurologic disorders. In this review, we have summarized the various modes of iN-PBMT delivery and their application in the treatment of brain disorders.

\section{Key words}

Photobiomodulation; Intranasal; Laser; Low-level lasers 


\section{INTRODUCTION}

Photobiomodulation therapy (PBMT), also known as low-level laser therapy (LLLT), is widely used to treat various medical conditions such as pain, inflammation, blood disorders, musculoskeletal conditions, as well as tissue regeneration. ${ }^{1}$ PBMT has steadily widened the range of applications to the brain diseases: neurotrauma, neurodegenerative diseases, and neuropsychiatric disorders., ${ }^{2,3}$ Moreover, brain PBMT is an effective therapeutic modality for central nervous system (CNS) disorders. ${ }^{4}$ Through several studies, it is proven that PBMT can improve cerebral blood flow, metabolic activity, neurogenesis, and neuroprotection by antioxidant and anti-inflammatory pathway activation. ${ }^{5,6}$

To deliver the laser light in brain PBMT, several approaches were proposed: transcranial, ${ }^{7}$ intranasal, ${ }^{8}$ intraaural, ${ }^{9}$ and intraoral ${ }^{10}$ approaches. Among these approaches, intranasal PBMT (iN-PBMT) can overcome the limitations of the PBMT to provide effective irradiation to the brain, especially on the limbic structures and prefrontal areas. ${ }^{11}$

In this review, we summarized the applicability of different intranasal irradiation approaches: from the nostrilbased method to the implanted nasal methods. Evidence for the effectiveness of iN-PBMT for neurological, neurodegenerative, and neuropsychiatric disorders will also be reviewed.

\section{INTRANASAL PHOTOBIOMODULATION THERAPY}

\section{From the nostril}

The nose serves as a direct pathway to the brain. Thus, nasal administration could be an effective, efficient and non-invasive option for delivering therapeutic materials to the CNS (Fig. 1A). ${ }^{12}$ Several intranasal portable devices for iN-PBMT through the nostril approach become commercially available. These iN-PBMT devices are both with red and near-infrared (NIR) diodes $(600-680 \mathrm{~nm}$ and $800-$ $850 \mathrm{~nm}$ wavelength).

It is known that iN-PBMT through the nostril approach improves oxygenation and leads to increased adenosine triphosphate levels in various tissues, including the brain. PBMT absorbed by blood leads to partial photochemical dissociation of hemoglobin ligand complexes such as oxygen, carbon dioxide, nitric oxide (NO). ${ }^{13-15}$ As a result of light-induced photodissociation of oxyhemoglobin, decreased in arterial oxygen saturation $\left(\mathrm{SpO}_{2}\right)$ in blood capillary vessels followed by significant enrichment of local tissue oxygenation occurs. ${ }^{16,17}$ With $660 \mathrm{~nm}$ wavelength photons, the laser irradiation effect can be amplified in lymphocytes. ${ }^{18}$ In addition, releasing small amounts of the NO, vasodilation and perfusion can be increased. Thus, oxygen delivery to tissues can be improved. Thus, nostrilbased iN-PBMT could be an effective and attractive modality for treatment of neurodegenerative, neuropsychiatric diseases, and ischemic brain injury. ${ }^{19}$ Nostril-based iN-PBMT could also modulate NO release from the endothelium and platelets, and can improve cerebrovascular
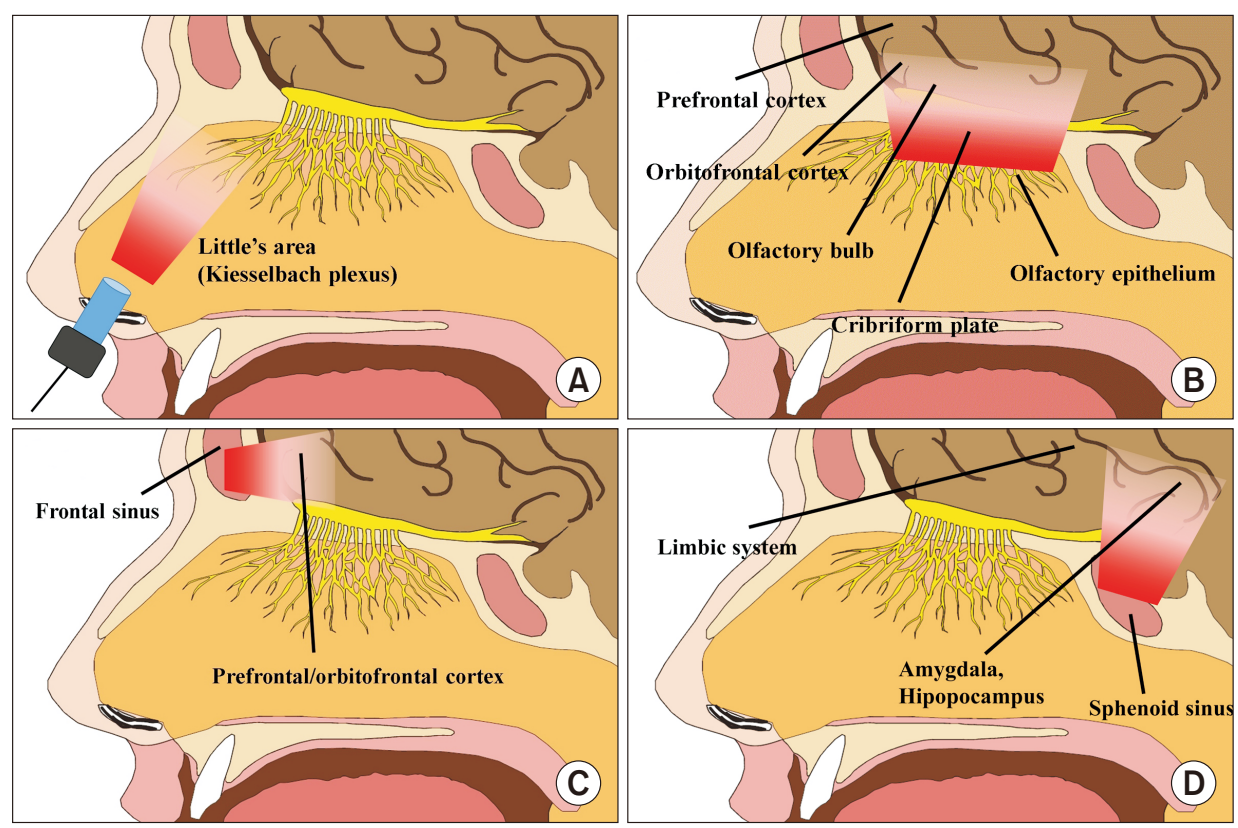

Fig. 1. Various approaches for intranasal photobiomodulation. (A) nostril-based approach or by (B) cribriform plate, (C) frontal sinus, (D) sphenoid sinus. 
circulation.

Several studies demonstrated that direct tissue exposure to red/NIR light can reduce the influx of polymorphonuclear leukocytes into a targeted site of inflammation, thus decreasing the oxidative burst. ${ }^{20,21}$ The remote neuroprotective action of iN-PBMT through the nostril approach could be related to modulation of reactive oxygen species (ROS) formation. ${ }^{22}$ The role of ROS is critical for reprogramming of macrophage polarization to M2 phenotype, and M2 macrophage releases anti-inflammatory mediators which are related to tissue recovery. ${ }^{23}$ In previous reports, $810 \mathrm{~nm}$ wavelength PBMT changed the macrophage polarization to an M2 phenotype and increased level of anti-inflammatory cytokines (interleukin (IL)-4, 13), ${ }^{24}$ but suppressed pro-inflammatory IL-6 ${ }^{25}$ resulting in alternative macrophage activation.

In addition, several other possible pathways were proposed that could mediate iN-PBMT: affecting the olfactory nerve, bulb, and endothelium, the autonomic nervous system, and the lymphatic system. ${ }^{26}$

\section{From the nasal submucosal space and nasal cav- ity}

As abovementioned, the nostril approach through portable devices only provides a negligible amount of light energy into the deep brain structures. ${ }^{11}$ Thus, recently, implantable iN-PBMT devices were developed to overcome these limitations. With a simple procedure, miniature light-emitting diodes (LEDs) can be implanted into the submucosal pockets in accessible areas of the nose.

The olfactory epithelium is an epithelial tissue layer in the nasal cavity. It is defined by the cribriform plate, which separates the nasal cavity from the brain. As it is close to the cribriform plate, it is proposed as possible and effective implant location for iN-PBMT device. If the implantable light device is located at the cribriform plate, the ventromedial cortex (PFC) and the olfactory bulb could be effectively irraidated (Fig. 1B). Intranasal delivery of red/NIR light can illuminate anteromedial and posteromedial portions of the orbitofrontal cortex (OFC). In addition, when the light source is positioned proximal to the cribriform plate, deposition of the energy on the ventromedial PFC is 658- and 46-fold greater than when the light source is implanted in the nostril or the middle of the nose, respectively. ${ }^{11}$ In addition, comparing with the dorsolateral PFC, the light source positioned in the cribriform plate led a higher light influence on the ventromedial PFC and OFC. In addition, limbic structures lamygdala and hippocampus) receive only negligible energy from a light source from the nostril, both the cribriform plate and the mid-nose locations can allow a higher deposition of light. $^{11}$

PFC and OFC can be also effectively irradiated when the implantable light device is inserted at the frontal sinus (Fig. 1C).

\section{From the sphenoid sinus}

In the sphenoid sinus, an implantable optical fiber can be positioned (Fig. 1D). For instance, the tip of the optical fiber connected to a LED sourcecan be placed as an indwelling device through endoscopic visualization in the sphenoid sinus. ${ }^{27}$ However, this requires a surgical procedure to place the device in a fragile bone structure. The sphenoidal sinus lies adjacent to important limbic system structures such as the pituitary gland, amygdala, hypothalamus, and hippocampus. The amygdala is located anterior to the hippocampus and medially in the temporal lobes. The amygdala resides lateral to and somewhat posterior to the sphenoid sinus. In general, the amygdala processes emotional responses (perception of facial expression, anxiety, fear, memory, aggression, decision making..$^{28}$ Adjacent to the pituitary gland on either side of the third ventricle and above/posterior to the sphenoid sinus, the hippocampus is located. It is involved in the regulation of endocrine, body temperature, food water intake, reproduction and sexual behavior, circadian rhythms, fatigue, emotional responses, and memory function. The hippocampus is a convex structure composed of gray matter tissue inside the para-hippocampal gyrus. It mediates several higher cognitive functions le.g., learning, long-term memory, spatial navigation, regulation of hypothalamic functions, and emotions). ${ }^{29}$

The sphenoid sinus could be used as a proper location of an implanted light source. It could provide therapeutic amounts of light to the limbic structures (Fig. 1D). A study made an effort to experimentally examine the light delivery and photon distribution from a transsphenoidal approach, in order to achieve sufficient irradiation of the pars compacta of substantia nigra (SN) in a human cadaver. ${ }^{10}$ In this study, authors coupled an optical fiber-based light diffuser to a laser diode emitting different wavelengths of 671 or $808 \mathrm{~nm}$, and then the probe was placed in the sphenoidal sinus towards the pars compacta of $\mathrm{SN}$ under endoscopic guidance. In compacta of SN, about $0.36 \%$ of $808 \mathrm{~nm}$ wavelength and $0.03 \%$ of $671 \mathrm{~nm}$ wavelength light emitted by the optical fiber could be reached. When sufficient power was used, these delivered light could provide a sufficient poststimulatory fluence to the SN. In motor planning, movement and reward-seeking, the SN plays an important role. 
With transsphenoidal illumination, a reasonable fraction of the light energy could be deposited in the amygdala, hypothalamus, hippocampus, and pituitary gland, before reaching the $\mathrm{SN}$.

\section{INTRANASAL PHOTOBIOMODULATION THERAPY FOR BRAIN CONDITIONS}

\section{Neurodegenerative disorders}

In one recent study, rapid reversal of cognitive decline and olfactory dysfunction in a mild cognitive impairment (MCI) patient after PBMT via transcranial and intranasal approach was reported. ${ }^{2}$ Cognitive enhancement was observed by improvements in executive function/visuospatial ability, mathematical ability, and orientation. Working memory and attention also showed significant improvements in the patient.

In addition, some researchers have reported the potential neurotherapeutic role of iN-PBMT for Alzheimer's disease (AD) and Parkinson's disease (PD). In a recent case series, 3-months of transcranial plus intranasal PBMT significantly enhanced cognitive performance in patients with mild to moderately severe dementia. ${ }^{8}$ With a wearable transcranial device, an intranasal LED applicator (810 $\mathrm{nm}$ wavelength) was applied providing a $13.8 \mathrm{~J} / \mathrm{cm}^{2}$ to the nasal cavity. Besides the improvement in cognitive abilities as assessed by the Mini-Mental State Examination (MMSE) and Alzheimer's Disease Assessment Scale (ADAS-cog), increased sleep quality and decreased levels of anxiety, anger outbursts, and wandering were also observed. In a recent study, Vielight Neuro Gamma device (Vielight Inc., Canada) was used in four patients diagnosed with dementia or $\mathrm{AD} .{ }^{30}$ With a transcranial headset device, the intranasal LED applicator $(810 \mathrm{~nm}$ wavelength, $40-\mathrm{Hz}$ pulse wave, $15 \mathrm{~J} / \mathrm{cm}^{2}$ ) was applied to the nasal cavity for 3 days/week for 12 weeks at home. Cognitive and behavioral functions were improved according to the neuropsychiatric inventory scores and the ADA-cog score. With multimodality PBMT, cerebral perfusion and connectivity between the lateral parietal nodes in the default-mode network (DMN) and the posterior cingulate cortex.

Some researchers have tested the therapeutic role of iN-PBMT for PD patients. ${ }^{31,32}$

\section{Traumatic/ischemic brain injury}

In a randomized, double-blind, sham-controlled pilot trial study in eight veterans with mild-TBI, combined application of the transcranial LED helmet and intranasal LED applicator for 2 days/week for 8 weeks, resulted in significant improvements in attention and executive func- tion as well as sleep quality at 1-week post-treatment. ${ }^{33}$

In one study, intranasal intranasal laser irradiation (10 days, 30 minutes, $650 \mathrm{~nm}, 8.38 \mathrm{~mW} / \mathrm{cm}^{2}$ ) showed improvement of aggregation of red blood cells, plasma viscosity of blood, and lipid profiles. ${ }^{34}$

\section{Neuropsychiatric disorders and insomnia}

Recently, long-term PBMT showed the overall therapeutic effect in a patient with major depression and anxiety. ${ }^{35}$ For the first 22 months, single-modality iN-PBMT (810 nm LED) was applied to both nostrils providing a $10.65 \mathrm{~J} / \mathrm{cm}^{2}$ per nostril. With the progressive increase of the frequency of iN-PBMT sessions, the anxiety symptoms showed regression with about a 3-fold reduction in Anxiety Symptoms Questionnaire Scores. However, iNPBMT alone did not improve the depressive symptoms until an additional trancranial laser irradiation to the forehead. According to their speculation, the systemic effects of iN-PBMT via the blood cells possibly contributed to the observed anxiolytic effect.

There is also proof of the therapeutic application of PBMT in sleep disorders. Red-light irradiation $1658 \mathrm{~nm}$, $30 \mathrm{~J} / \mathrm{cm}^{2}$ ) for two weeks using a whole body treatment machine has been shown to improve sleep quality and serum melatonin levels. ${ }^{36}$ In studies of the application of tPBMT in TBI patients, improvement in sleep was reported by many of the patients. ${ }^{37,38} \mathrm{~A}$ combined transcranial and intranasal PBMT also resulted in better sleep in patients diagnosed with $\mathrm{AD}$ and $\mathrm{TBI} .{ }^{8,33}$ According to a previous report, sleepiness is a common side-effect following the use of $10-\mathrm{Hz}$ intranasal portable devices, inducing brain alpha wave $(8-12 \mathrm{~Hz})$, while also producing neuronal stimulation. ${ }^{2}$ Although mechanisms of action involved in PBMT for sleep improvement are still unknown, modulation of circadian rhythms via an increase in serum melatonin level ${ }^{36,39}$ and stimulation of systemic homeostatic response via the blood circulatory system ${ }^{40}$ were proposed as possible mechanisms.

\section{CONCLUSIONS}

Table 1 summarizes the results of some clinical studies on the effects of iN-PBMT on various brain conditions. While iN-PBMT through nostrils for wellness is available, its efficacy and effectiveness is still unproven. Submucosal and frontal iN-PBMT has potential as a novel therapeutic modality, however, there are no aviailable device is in the market. Other approaches through the deeper nose (cribriform plate or sphenoid sinus), iN-PBMT is not yet available with current technology. However, iN-PBMT, 
Table 1. Intranasal photobiomodulation therapy (PBMT) for brain conditions

\begin{tabular}{|c|c|c|c|c|}
\hline Application & & Study design & Intranasal PBMT condition & References \\
\hline \multirow[t]{4}{*}{ Neurodegenerative disorders } & Mild cognitive impairment & Case report & $810 \mathrm{~nm}$ wavelength $10-\mathrm{Hz}$ pulsed wave & Salehpour et al. $^{2}$ \\
\hline & Dementia & Case series & $810 \mathrm{~nm}$ wavelength $10-\mathrm{Hz}$ pulsed wave & Saltmarche et al. ${ }^{8}$ \\
\hline & & Case series & $810 \mathrm{~nm}$ wavelength $10-\mathrm{Hz}$ pulsed wave & $\mathrm{Chao}^{30}$ \\
\hline & Parkinson's disease & $\begin{array}{l}\text { Prospective proof-of-concept } \\
\text { study }\end{array}$ & $810 \mathrm{~nm}$ wavelength $50-\mathrm{Hz}$ pulsed wave & Liebert et al. ${ }^{32}$ \\
\hline \multirow[t]{2}{*}{ Traumatic/ischemic brain injury } & Mild traumatic brain injury & $\begin{array}{l}\text { Randomized, double-blind, } \\
\text { sham-controlled pilot trial study }\end{array}$ & $810 \mathrm{~nm}$ wavelength $10-\mathrm{Hz}$ pulsed wave & Bogdanova et al. $^{33}$ \\
\hline & Cerebral infarction & $\begin{array}{l}\text { Randomized, double-blind, } \\
\text { placebo-controlled study }\end{array}$ & $650 \mathrm{~nm}$ wavelength & Liu et al. ${ }^{34}$ \\
\hline Neuropsychiatric disorders & Major depression, anxiety & Case report & $810 \mathrm{~nm}$ wavelength $10-\mathrm{Hz}$ pulsed wave & Caldieraro et al..$^{35}$ \\
\hline
\end{tabular}

using LED applicators, has potential as a novel approach for neurorehabilitation. Thus, more future studies comparing sham, and transcranial PBMT are also warranted.

\section{CONFLICT OF INTEREST}

No potential conflict of interest relevant to this article was reported.

\section{FUNDING}

This research was supported by Basic Science Research Program through the National Research Foundation of Korea (NRF) funded by the Ministry of Education (2021R1I1A1A01052298).

\section{REFERENCES}

1. Chung H, Dai T, Sharma SK, Huang YY, Carroll JD, Hamblin MR. The nuts and bolts of low-level laser (light) therapy. Ann Biomed Eng 2012;40:516-33.

2. Salehpour F, Hamblin MR, DiDuro JO. Rapid reversal of cognitive decline, olfactory dysfunction, and quality of life using multimodality photobiomodulation therapy: case report. Photobiomodul Photomed Laser Surg 2019;37:159-67.

3. Chan AS, Lee TL, Yeung MK, Hamblin MR. Photobiomodulation improves the frontal cognitive function of older adults. Int J Geriatr Psychiatry 2019;34:369-77.

4. Fitzgerald M, Hodgetts S, Van Den Heuvel C, Natoli R, Hart NS, Valter $\mathrm{K}$, et al. Red/near-infrared irradiation therapy for treatment of central nervous system injuries and disorders. Rev Neurosci 2013;24:205-26.

5. Grillo SL, Duggett NA, Ennaceur A, Chazot PL. Non-invasive infra-red therapy (1072 $\mathrm{nm}$ ) reduces $\beta$-amyloid protein levels in the brain of an Alzheimer's disease mouse model, TASTPM. J Photochem Photobiol B 2013;123:13-22.

6. Hamblin MR. Shining light on the head: photobiomodulation for brain disorders. BBA Clin 2016;6:113-24.

7. Thunshelle C, Hamblin MR. Transcranial low-level laser (Light) therapy for brain injury. Photomed Laser Surg 2016;34:587-98.

8. Saltmarche AE, Naeser MA, Ho KF, Hamblin MR, Lim L. Significant improvement in cognition in mild to moderately severe dementia cases treated with transcranial plus intranasal photobiomodulation: case series report. Photomed Laser Surg 2017;35:432-41.

9. Sun L, Peräkylä J, Kovalainen A, Ogawa KH, Karhunen PJ, Hartikainen KM. Human brain reacts to transcranial extraocular light. PLoS One 2016;11:e0149525.

10. Pitzschke A, Lovisa B, Seydoux O, Zellweger M, Pfleiderer M, Tardy Y, et al. Red and NIR light dosimetry in the human deep brain. Phys Med Biol 2015;60:2921-37.

11. Cassano P, Tran AP, Katnani H, Bleier BS, Hamblin MR, Yuan $Y$, et al. Selective photobiomodulation for emotion regulation: model-based dosimetry study. Neurophotonics 2019;6:015004.

12. Hanson LR, Frey WH 2nd. Intranasal delivery bypasses the blood-brain barrier to target therapeutic agents to the central nervous system and treat neurodegenerative disease. BMC Neurosci 2008;9(Suppl 3):S5.

13. Komorowska M, Cuissot A, Czarnołeski A, Białas W. Erythrocyte response to near-infrared radiation. J Photochem Photobiol B 2002;68:93-100.

14. Vladimirov YA, Osipov AN, Klebanov GI. Photobiological principles of therapeutic applications of laser radiation. Biochemistry (Mosc) 2004:69:81-90

15. Lohr NL, Keszler A, Pratt P, Bienengraber M, Warltier DC, Hogg N. Enhancement of nitric oxide release from nitrosyl hemoglobin and nitrosyl myoglobin by red/near infrared radiation: potential role in cardioprotection. J Mol Cell Cardiol 2009;47:256-63.

16. Asimov MM, Korolevich AN, Konstantinova EÉ. Kinetics of oxygenation of skin tissue exposed to low-intensity laser radiation. J Appl Spectrosc 2007;74:133-9.

17. Yesman SS, Mamilov SO, Veligotsky DV, Gisbrecht Al. Local changes in arterial oxygen saturation induced by visible and 
near-infrared light radiation. Lasers Med Sci 2016;31:145-9.

18. Stadler I, Evans R, Kolb B, Naim JO, Narayan V, Buehner N, et al. In vitro effects of low-level laser irradiation at $660 \mathrm{~nm}$ on peripheral blood lymphocytes. Lasers Surg Med 2000;27:255-61.

19. Chrapko W, Jurasz P, Radomski MW, Archer SL, Newman SC, Baker $\mathrm{G}$, et al. Alteration of decreased plasma NO metabolites and platelet NO synthase activity by paroxetine in depressed patients. Neuropsychopharmacology 2006;31:1286-93.

20. de Lima FM, Villaverde AB, Albertini R, Corrêa JC, Carvalho RL, Munin E, et al. Dual Effect of low-level laser therapy (LLLT) on the acute lung inflammation induced by intestinal ischemia and reperfusion: action on anti- and pro-inflammatory cytokines. Lasers Surg Med 2011;43:410-20.

21. Oliveira MC Jr, Greiffo FR, Rigonato-Oliveira NC, Custódio RW, Silva VR, Damaceno-Rodrigues NR, et al. Low level laser therapy reduces acute lung inflammation in a model of pulmonary and extrapulmonary LPS-induced ARDS. J Photochem Photobiol B 2014;134:57-63.

22. Karu TI, Pyatibrat LV, Afanasyeva NI. Cellular effects of low power laser therapy can be mediated by nitric oxide. Lasers Surg Med 2005;36:307-14.

23. Zhang Y, Choksi S, Chen K, Pobezinskaya Y, Linnoila I, Liu ZG. ROS play a critical role in the differentiation of alternatively activated macrophages and the occurrence of tumor-associated macrophages. Cell Res 2013;23:898-914.

24. Song JW, Li K, Liang ZW, Dai C, Shen XF, Gong YZ, et al. Lowlevel laser facilitates alternatively activated macrophage/ microglia polarization and promotes functional recovery after crush spinal cord injury in rats. Sci Rep 2017;7:620.

25. Byrnes KR, Waynant RW, Ilev IK, Wu X, Barna L, Smith K, et al. Light promotes regeneration and functional recovery and alters the immune response after spinal cord injury. Lasers Surg Med 2005;36:171-85.

26. Liu TCY, Wu DF, Gu ZQ, Wu M. Applications of intranasal low intensity laser therapy in sports medicine. J Innov Opt Health Sci 2010;03:1-16.

27. Wiebracht ND, Zimmer LA. Complex anatomy of the sphenoid sinus: a radiographic study and literature review. J Neurol Surg B Skull Base 2014;75:378-82.

28. Sah P, Faber ES, Lopez De Armentia M, Power J. The amygdaloid complex: anatomy and physiology. Physiol Rev 2003;83:803-34.

29. Anand KS, Dhikav V. Hippocampus in health and disease: an overview. Ann Indian Acad Neurol 2012;15:239-46.

30. Chao LL. Effects of home photobiomodulation treatments on cognitive and behavioral function, cerebral perfusion, and resting-state functional connectivity in patients with dementia: a pilot trial. Photobiomodul Photomed Laser Surg 2019;37:13341.

31. Johnstone DM, Moro C, Stone J, Benabid AL, Mitrofanis J. Turning on lights to stop neurodegeneration: the potential of near infrared light therapy in Alzheimer's and Parkinson's disease. Front Neurosci 2016;9:500.

32. Liebert A, Bicknell B, Laakso EL, Heller G, Jalilitabaei P, Tilley S, et al. Improvements in clinical signs of Parkinson's disease using photobiomodulation: a prospective proof-of-concept study. BMC Neurol 2021;21:256.

33. Bogdanova Y, Ho V, Martin P, Ho M, Yee M, Hamblin M, et al. Transcranial LED treatment for cognitive dysfunction and sleep in chronic TBI: randomized controlled pilot trial. Arch Phys Med Rehabil 2017;98:E122-3.

34. Liu TCY, Cheng L, Su WJ, Zhang YW, Shi Y, Liu AH, et al. Randomized, double-blind, and placebo-controlled clinic report of intranasal low-intensity laser therapy on vascular diseases. Int J Photoenerg 2012;2012:489713.

35. Caldieraro MA, Sani G, Bui E, Cassano P. Long-term nearinfrared photobiomodulation for anxious depression complicated by takotsubo cardiomyopathy. J Clin Psychopharmacol 2018;38:268-70.

36. Zhao J, Tian Y, Nie J, Xu J, Liu D. Red light and the sleep quality and endurance performance of Chinese female basketball players. J Athl Train 2012;47:673-8.

37. Naeser MA, Saltmarche A, Krengel MH, Hamblin MR, Knight JA. Improved cognitive function after transcranial, light-emitting diode treatments in chronic, traumatic brain injury: two case reports. Photomed Laser Surg 2011;29:351-8.

38. Morries LD, Cassano P, Henderson TA. Treatments for traumatic brain injury with emphasis on transcranial near-infrared laser phototherapy. Neuropsychiatr Dis Treat 2015;11:2159-75.

39. Liu TCY, Wu DF, Gu ZQ, Wu M. Applications of intranasal low intensity laser therapy in sports medicine. J Innov Opt Health Sci 2010;03:1-16.

40. Moshkovska T, Mayberry J. It is time to test low level laser therapy in Great Britain. Postgrad Med J 2005;81:436-41.

How to cite this article: Yoo SH. Intranasal photobiomodulation therapy for brain conditions: a review. Med Lasers 2021;10:132-137. https://doi.org/10.25289/ML.2021.10.3.132 